\title{
A novel method for sensing rotational speed, linear displacement and current using superconducting BPSCCO magnetic sensor
}

\author{
T K DEY*, A RAY and S K GHATAK \\ Cryogenic Engineering Centre, 'Department of Physics and Meteorology, Indian Institute of Technology, \\ Kharagpur 721 302, India
}

\begin{abstract}
For many decades, magnetic sensors have been of great assistance to mankind in variety of functions that include simple compass based navigational systems to devices that monitor the invisible biological activities. In industries magnetic sensors are in great demand for control and measurement of linear and rotary position sensing etc, because of its non destructive and contact less way of detection. Consequently, newer, smarter and cheaper materials are continuously being explored to suit the varied needs of technological requirements. In the present communication, the characteristics of a magnetic sensor, based on the non linear electromagnetic response of the weak links present in the polycrystalline BPSCCO superconductor are reported. The second harmonic response of sintered superconducting BPSCCO pellet in an alternating magnetic field at $40 \mathrm{kHz}$ and $77 \mathrm{~K}$ being a strong linear function of low d.c. magnetic field has been utilized for the development of highly sensitive magnetic field sensors. The noise limited resolution of the sensor is found to be $3.16 \times 10^{-9} \mathrm{~T} / \sqrt{\mathrm{Hz}}$ for $H_{\text {a.c. }}=16$ Oe and frequency $40 \mathrm{kHz}$. We further demonstrate that such HTSC based magnetic sensors are capable of sensing the rotational speed, small displacement and direct current with good resolution. The experimental methods and results obtained are discussed.
\end{abstract}

Keywords. Magnetic sensor; superconductor; rotational speed sensor; displacement sensor.

\section{Introduction}

The sensors those are in great demand in both the basic research and technological development have been developed to detect physical quantities like pressure, temperature, current, velocity (tachometer, flow meter etc), magnetic field and displacement among many other applications. Magnetic sensors are often preferred in automotive and industrial environments, because they offer several key advantages, viz. contact less measurements of mechanical quantities like angle of rotation, angular speed, and displacement etc. They are robust and inexpensive to fabricate. A majority of these sensors are based on the Hall effect, inductive effect and now-a-days anisotropic magnetoresistance (AMR) and giant magnetoresistance (GMR) effect. However, high temperature superconductors (HTSC) also keep their potential to be used as magnetic sensors. In presence of an alternating magnetic field of frequency $(f)$, the polycrystalline HTSCs generate only the odd harmonics of magnetic susceptibility as coming out of their symmetric magnetization. However, superposition of a small d.c. field breaks this symmetry and brings the appearance of even harmonics. Amplitudes of the even harmonics are found to be a strongly linear function of applied d.c. field (Ghatak et al 1992; Khare et al 1997; Dey et al 1999).

\footnotetext{
*Author for correspondence
}

The origin of the harmonic generation and its modulation with d.c. magnetic field is explained elsewhere (Xenikos and Lamberger 1990; Muller 1990; Qin and Ong 1999). The observed linear dependence of the amplitude of the second harmonic response with low d.c. magnetic field can be exploited as a highly sensitive magnetic field sensor (Gallop et al 1988, 1989; Khare et al 1997; Dey et al 1999). Magnetic sensors, with the capability of sensing very low magnetic fields are of great technical and commercial significance. Some of the potential areas of applications of such kind of magnetic sensors are the non-destructive evaluation as well as rotational speed, linear displacement, current and cryogen flow sensing. To date a great variety of rotational speed sensors have been developed. Nevertheless numerous problems of measurements still remain open (Kwa and Wolffenbuttel 1991). Recently, Li et al (2001) reported an improved MEMS based rotation sensor capable of detecting rotation speed up to $6000 \mathrm{rpm}$. Similarly, the position sensors, based on different operation mechanisms viz. magneto-resistive (Williumson 1968), Hall effect (Kano et al 1990), magneto-inductive (Vertesy et al 1990), capacitive (Moore et al 1993), eddy current (Vasseur and Villat 1994), have been reported. Current sensing, on the other hand plays an important role especially in automotive applications (Pross et al 1999) for power management applications. In the present communication, we report our results on the development of a novel, inexpensive and highly sensitive high $T_{\mathrm{c}}$ based magnetic sensor and 
Table 1. The lattice parameters $(a, b, c)$, density of the pellets and slopes of the best linear fit

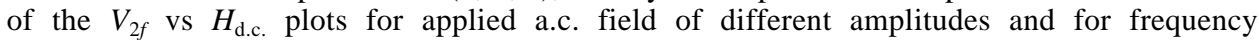
$f=40 \mathrm{kHz}$ for the three (Bi,Pb)-2223 samples. A (5.5 MPa), B (8 MPa) and C (11 MPa).

\begin{tabular}{|c|c|c|c|c|c|c|c|c|}
\hline \multirow[b]{2}{*}{ Sample } & \multirow[b]{2}{*}{$a$} & \multirow[b]{2}{*}{$b$} & \multirow[b]{2}{*}{$c$} & \multirow[b]{2}{*}{ Density $(\mathrm{g} / \mathrm{cc})$} & \multicolumn{4}{|c|}{$\delta V / \delta H_{\text {d.c. }}(\mathrm{mV} / \mathrm{Oe})$} \\
\hline & & & & & $H_{\text {a.c. }}=16 \mathrm{Oe}$ & $13 \mathrm{Oe}$ & $10 \mathrm{Oe}$ & $8 \mathrm{Oe}$ \\
\hline A & $5 \cdot 41$ & $5 \cdot 41$ & $37 \cdot 02$ & $5 \cdot 398$ & $0 \cdot 72$ & $1 \cdot 25$ & 1.92 & $3 \cdot 16$ \\
\hline B & $5 \cdot 4$ & $5 \cdot 4$ & $37 \cdot 01$ & 5.531 & $0 \cdot 24$ & 0.43 & 0.68 & 0.94 \\
\hline C & $5 \cdot 4$ & $5 \cdot 4$ & $37 \cdot 01$ & $5 \cdot 862$ & $0 \cdot 15$ & $0 \cdot 27$ & 0.41 & $0 \cdot 58$ \\
\hline
\end{tabular}

demonstrate its utility for rotational speed, displacement and current sensing. To the best of our knowledge, application of HTSC based magnetic sensor for rotational speed, displacement and current sensing is being reported for the first time.

\section{Materials and methods}

The BPSCCO sensor used in the present work was prepared from high quality $\mathrm{Bi}_{1 \cdot 6} \mathrm{~Pb}_{0.4} \mathrm{Sr}_{2} \mathrm{Ca}_{2} \mathrm{Cu}_{3} \mathrm{O}_{10+\delta}$ precursor powder obtained from M/s CAN Superconductors, Czech Republic. The said powder was cold pressed in the form of cylindrical pellets (dia. $\sim 12 \mathrm{~mm}$, thickness $\sim 4 \mathrm{~mm}$ ) with pressures of $5.5 \mathrm{MPa}, 8 \mathrm{MPa}$ and $11 \mathrm{MPa}$ and was subsequently sintered at $890^{\circ} \mathrm{C}$ for $6 \mathrm{~h}$ in air. The pellet was furnace cooled to room temperature slowly in air. $T_{\mathrm{c}}$, lattice parameters and the densities of these samples are listed in table 1. The experimental arrangements for characterization of the magnetic sensor are described elsewhere (Dey et al 1999).

\section{Results and discussion}

\subsection{Magnetic field sensing using superconducting BPSCCO}

Figure 1 shows the experimental data in the range 0 $<H_{\text {d.c. }}<4 \mathrm{G}$ and the corresponding solid lines represent the best-fit linear relation. The slopes $\left(\mathrm{d} V_{2 f} / \mathrm{dH}_{\text {d.c. }}\right)$ of these linear fits are given in table 1 . The rate of increase in the response is found to be higher for higher a.c. field amplitude and higher frequencies. This rate also increases as the density of the sample decreases. The field noise $\left(\sqrt{S_{\mathrm{B}}}\right)$, or the smallest field change, which could be detected by the sensor, is estimated from,

$$
\sqrt{S_{\mathrm{B}}}=\left[\Delta V /\left(\delta V / \delta H_{\text {d.c. }}\right)\right],
$$

where, $\Delta V$ is the voltage noise at the output of the lock-in amplifier and $\left(\delta V / \delta H_{\text {d.c. }}\right)$ the transfer function of the sensor. The value of $\left(\delta V / \delta H_{\text {d.c. }}\right)$ is known from the slope of the linear region of $V_{2 f}$ vs $H_{\text {d.c. }}$ plots. The highest noise sensitivity $\left(\sqrt{S_{\mathrm{B}}}\right)$ for our BPSCCO sensor (A) was found to be $3 \cdot 16 \times 10^{-9} \mathrm{~T} / \sqrt{\mathrm{Hz}}$. This figure may

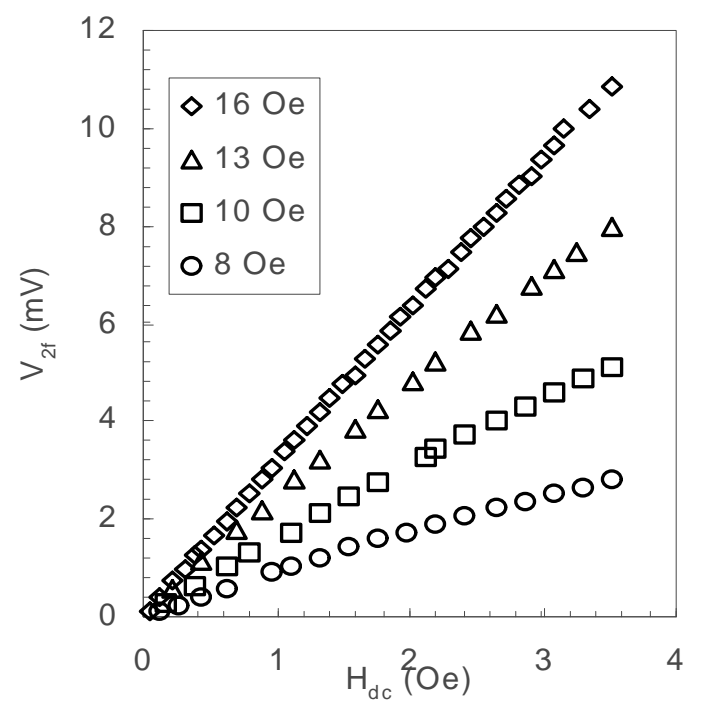

Figure 1. Second harmonic response of the $(\mathrm{Bi}, \mathrm{Pb})-2223$ magnetic sensor as a function of d.c. field between 0 and $4 \mathrm{G}$ at $40 \mathrm{kHz}$ for different a.c. field amplitudes: $H_{\text {a.c. }}=8,10,13$ and 16 Oe.

be compared with the estimated theoretical limit of $\sim 10^{-10} \mathrm{~T} / \sqrt{\mathrm{Hz}}$ (Gallop et al 1989).

\subsection{Sensor as rotational speed sensor}

Figure 2(a) shows the experimental arrangement for measuring the rotational speed of a commercial tape recorder motor. A wheel is connected to the motor, over which two small pieces of permanent magnets are attached at the periphery at diametrically opposite points. For every rotation of the wheel, the magnetic flux cuts the sensor twice. A pick up coil (50 turns, 46 swg enameled copper wire) is wound directly on the sample. The second harmonic amplitude of the applied frequency at the primary a.c. coil, induced in the pick up coil is detected by a lock-in-amplifier (Stanford, model SR 830). The output of the lock-in-amplifier is connected to a digital oscilloscope (HP, model 54502A). A frequency counter connected parallel to the oscilloscope reads twice the frequency of oscillation of the $2 f$ signal generated in the magnetic sensor. The frequency of rotation of the motor was calibrated using an LDR. 
Figures 2(c) and (d) show the typical oscilloscope trace of the output of the lock-in amplifier corresponding to frequencies $750 \mathrm{rpm}$ and $1980 \mathrm{rpm}$. Since the distribution and intensity of the magnetic field lines from the magnet pieces was not exactly identical, pure square wave pattern was not observed. It may be seen from figure 2(b) that the rpm estimated using the $2 f$ signal when plotted with the calibrated speed of rotation of the motor, results in a linear plot with a slope of $45^{\circ}$ which proves the exact correspondence between the actual speed of rotation of the motor and that obtained using the present $2 f$ based HTSC magnetic sensor.

\subsection{Sensor as a displacement sensor}

The experimental set-up for the measurement of small displacement of a ferrite piece relative to the position of the sensor is shown in figure 3(a). The object that undergoes displacement is attached with a small piece of permanent magnet. As the proximity of the object and the sensor is closer, the density of magnetic flux that crosses the sensor increases. This in turn increases the induced second harmonic signal. Figure $3 \mathrm{~b}$ shows the measured $2 f$ amplitude when the object is vertically moved slowly away from the sensor. A linear response between the displacement and $2 f$ is clearly evident up to $\sim 3 \mathrm{~mm}$. However, beyond this, the field profile of the magnet becomes nonlinear and the intensity rapidly decreases and the $2 f$ signal becomes hardly detectable. The sensitivity of the present HTSC displacement sensor is close to $1 \mu \mathrm{m}$.

\subsection{Direct current sensing with the magnetic sensor}

For the current sensing application, the current to be detected is allowed to pass through a small solenoid with 200 turns of $40 \mathrm{swg}$ copper wire kept just below the liquid $\mathrm{N}_{2}$ dewar as shown in figure 4(a). The passage of direct current through the monitoring solenoid causes the generation of d.c. magnetic field and the sensor in turn generates the $2 f$ signal. A linear response of the $2 f$ amplitude as a function of sensing current is as shown in
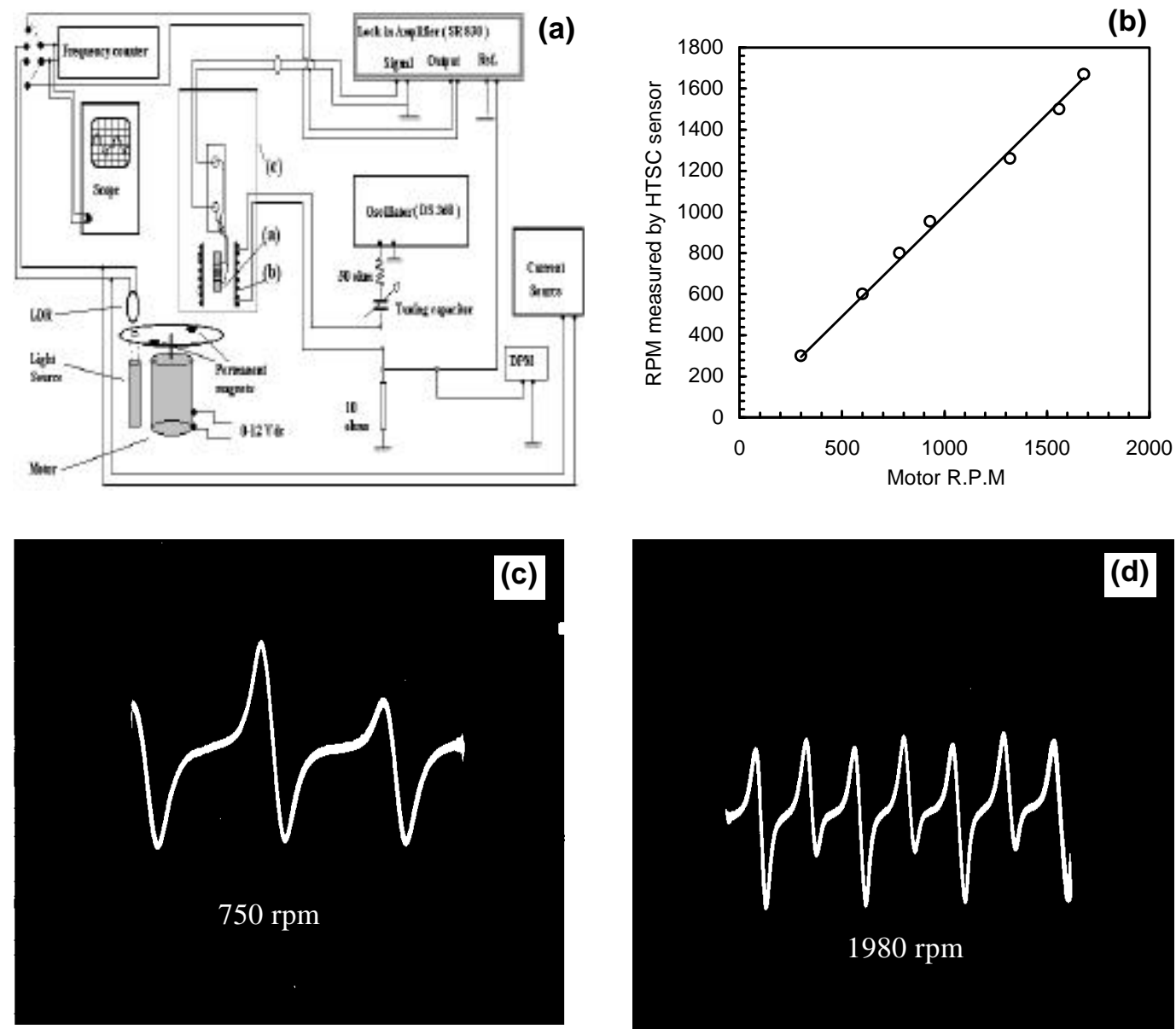

Figure 2. (a) Experimental arrangement for the measurement of rotational speed of a motor, (b) experimental data showing the correspondence between the rpm measured by the superconductive magnetic sensor with that of the calibrated values using LDR, typical oscilloscope trace of the $2 f$ amplitude output of the lock-in amplifier corresponding to (c) $750 \mathrm{rpm}$ and (d) $1980 \mathrm{rpm}$ of the rotating motor as detected by the sensor. 

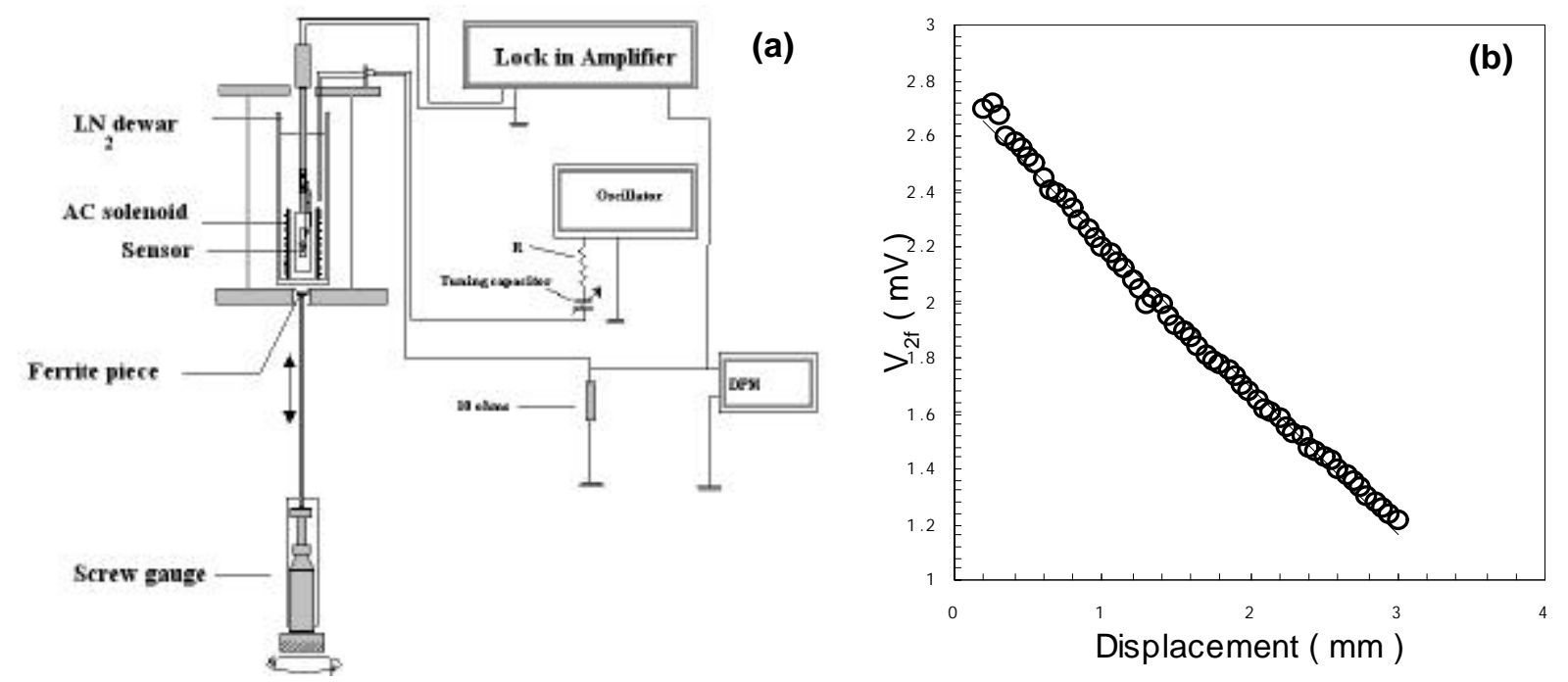

Figure 3. (a) Experimental arrangement for the measurement of linear displacement and (b) second harmonic voltages of the superconductive magnetic sensor as function of small displacements.
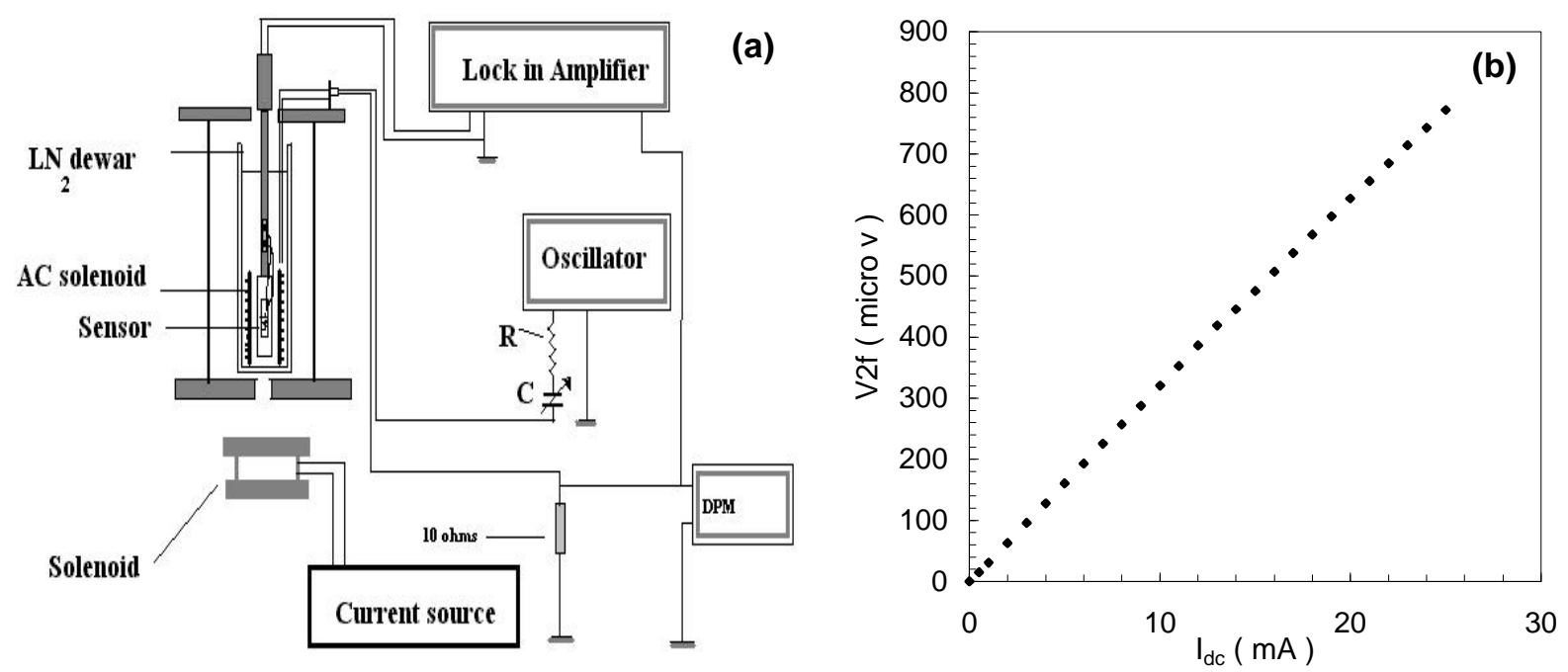

Figure 4. (a) Experimental arrangement for current sensing by a superconducting $2 f$ based magnetic sensor and (b) the variation of the second harmonic voltage as a function of the current flowing through the solenoid.

figure 4(b). The sensitivity of our present sensor is found to be $31.4 \mu \mathrm{V} / \mathrm{mA}$. However, this sensitivity could be controlled to higher figure by using the solenoid with higher number of turns connected to current measuring circuit. This would increase the magnetic flux density crossing the sample and hence the $2 f$ signal.

\section{Conclusions}

An HTSC based magnetic sensor capable of sensing the rotational speed, displacement and current is reported. The sensor operates at liquid $\mathrm{N}_{2}$ temperature and having the noise sensitivity of $\sim 3.6 \times 10^{-9} \mathrm{~T} / \sqrt{\mathrm{Hz}}$. The sensor is capable of measuring rotational speed up to $2000 \mathrm{rpm}$
$( \pm 1 \mathrm{rpm})$ and small displacement in the range from $\sim 0$ and $3 \mathrm{~mm}$ and the direct current in any circuit.

\section{Acknowledgement}

One of the authors (AR) is grateful to the Department of Science and Technology, New Delhi, for providing financial assistance in the form of a Senior Research Fellowship.

\section{References}

Dey T K, Chattopadhyay M K and Ghatak S K 1999 Sensors and Actuators $\mathbf{B 5 5} 222$ 
Gallop J C, Lilleyaman S, Langham C D, Redcliffe W J, Gee M and Stewart M 1988 Cryogenics 28691

Gallop J C, Lilleyaman S, Langham C D, Redcliffe W J and Stewart M 1989 IEEE Trans. Magn. 25896

Ghatak S K, Mitra A K and Sen D 1992 Phys. Rev. B45 951

Kano Y, Hasebe S, Huang C, Yamada T and Inubuse M 1990 IEEE Trans. Magn. 262023

Khare N, Gupta A K, Srivastava S K, Khare S and Padmanaban V P N 1997 Meas. Sci. Technol. 829

Kwa T A and Wolffenbuttel R F 1991 IEEE Transducers 24

Li Wen J, Mei Tao and Sun W 2001 Sensors and Actuators A89 56
Moore T D, Turner S and Speake C C 1993 Meas. Sci. Technol. 41519

Muller K H 1990 Physica C168 585

Pross A, Lewis C and Hesketh T 1999 Sensors and Actuators A76 72

Qin M J and Ong C K 1999 Phys. Rev. B61 9786

Vasseur P and Villat A 1994 Meas. Sci. Technol. 5889

Vertesy G, Szollosy J and Lovas A 1990 IEEE Trans. Magn. 26 2026

Williumson W J 1968 IEEE Trans. Magn. 4162

Xenikos D G and Lamberger T R 1990 Phys. Rev. B41 869 